\title{
Analytic Approximation of Solutions of Parabolic Partial Differential Equations with Variable Coefficients
}

\author{
Vladislav V. Kravchenko, Josafath A. Otero, and Sergii M. Torba \\ Departamento de Matemáticas, Unidad Querétaro, CINVESTAV del IPN, Libramiento Norponiente No. 2000, \\ Fracc. Real de Juriquilla, 76230 Querétaro, QRO, Mexico \\ Correspondence should be addressed to Sergii M. Torba; storba@math.cinvestav.edu.mx
}

Received 20 June 2017; Accepted 3 August 2017; Published 14 November 2017

Academic Editor: Antonio Scarfone

Copyright (C) 2017 Vladislav V. Kravchenko et al. This is an open access article distributed under the Creative Commons Attribution License, which permits unrestricted use, distribution, and reproduction in any medium, provided the original work is properly cited.

A complete family of solutions for the one-dimensional reaction-diffusion equation, $u_{x x}(x, t)-q(x) u(x, t)=u_{t}(x, t)$, with a coefficient $q$ depending on $x$ is constructed. The solutions represent the images of the heat polynomials under the action of a transmutation operator. Their use allows one to obtain an explicit solution of the noncharacteristic Cauchy problem with sufficiently regular Cauchy data as well as to solve numerically initial boundary value problems. In the paper, the Dirichlet boundary conditions are considered; however, the proposed method can be easily extended onto other standard boundary conditions. The proposed numerical method is shown to reveal good accuracy.

\section{Introduction}

In the present work a complete system of solutions of a one-dimensional reaction-diffusion equation with a variable coefficient

$$
u_{x x}(x, t)-q(x) u(x, t)=u_{t}(x, t)
$$

considered on $\Omega:=(-b, b) \times(0, \tau)$ is obtained. We assume that the potential $q \in C[-b, b]$ may be complex valued. The completeness of the system is with respect to the uniform norm in the closed rectangle $\bar{\Omega}$. The system of solutions is shown to be useful for uniform approximation of solutions of initial boundary value problems for (1) as well as for explicit solution of the noncharacteristic Cauchy problem (see [1]) for (1) in terms of the formal powers arising in the spectral parameter power series (SPPS) method (see $[2,3])$. In the paper, the Dirichlet boundary conditions are considered; however the proposed method can be easily extended onto other standard boundary conditions.

The complete system of solutions is constructed with the aid of the transmutation operators relating (1) to the heat equation (see, e.g., [4-6]). The possibility of constructing complete systems of solutions by means of transmutation operators was proposed and explored in [4], and the approach developed in [4] requires the knowledge of the transmutation operators. In the present work using a mapping property of the transmutation operators discovered in [7] we show that the construction of the complete systems of solutions for equations of form (1), representing transmuted heat polynomials, can be realized with no previous construction of the transmutation operator. Moreover, the use of the mapping property leads to an explicit solution of the noncharacteristic Cauchy problem for (1) with Cauchy data belonging to a Holmgren class [1].

We illustrate the implementation of the complete system of the transmuted heat polynomials by a numerical solution of an initial boundary value problem for (1). The approximate solution is sought in the form of a linear combination of the transmuted heat polynomials and the initial and boundary conditions are satisfied by a collocation method. A remarkable accuracy is achieved in few seconds using MATLAB 2012 on a usual PC.

Besides this Introduction the paper contains five sections. In Section 2 we recall the transmutation operators and some of their properties. In Section 3 an explicit solution of the noncharacteristic Cauchy problem for (1) is obtained. In Section 4 we prove the completeness of the transmuted heat polynomials. In Section 5 the numerical method for solving 
initial boundary value problems for (1) implementing the transmuted heat polynomials is discussed. Section 6 presents a numerical illustration.

\section{Transmutation Operators and Formal Powers}

2.1. System of Recurrent Integrals. Let $q$ be a continuous complex valued function defined on the segment $[-b, b]$. Throughout the paper we suppose that $f$ is a nonvanishing solution of the equation

$$
f^{\prime \prime}-q(x) f=0
$$

on $(-b, b)$ such that $f(0)=1$ and $f^{\prime}(0)=\alpha$, where $\alpha$ is a complex number. In [3] the existence of such solution was proved.

Consider two sequences of recurrent integrals (see [2, 3]):

$$
\begin{aligned}
& X^{(0)} \equiv 1, \\
& X^{(n)}(x)=n \int_{0}^{x} X^{(n-1)}(s)\left(f^{2}(s)\right)^{(-1)^{n}} d s, \\
& \widetilde{X}^{(0)} \equiv 1, \\
& \widetilde{X}^{(n)}(x)=n \int_{0}^{x} \widetilde{X}^{(n-1)}(s)\left(f^{2}(s)\right)^{(-1)^{n-1}} d s, \\
& x \in[-b, b], n \in \mathbb{N} .
\end{aligned}
$$

Definition 1. The family of functions $\left\{\varphi_{k}\right\}_{k=0}^{\infty}$ constructed according to the rule

$$
\varphi_{k}(x)= \begin{cases}f(x) X^{(k)}(x), & k \text { odd } \\ f(x) \widetilde{X}^{(k)}(x), & k \text { even }\end{cases}
$$

is called the system of formal powers associated with $f$.

The formal powers arise in the spectral parameter power series (SPPS) representation for solutions of the onedimensional Schrödinger equation (see $[2,3])$.

2.2. The Transmutation Operator. For any $q \in C[-b, b]$ it is a well-known result $[5$, Chapter 1$]$ that there exists a function $(x, s) \mapsto K(x, s)$ defined on the domain $0 \leq|s| \leq|x| \leq b$, continuously differentiable, such that the equality

$$
A T v=T B v
$$

is valid for all $v \in C^{2}[-b, b]$, where $A:=\partial^{2} / \partial x^{2}-q, B:=$ $\partial^{2} / \partial x^{2}$, and $T$ has the form of a second-kind Volterra integral operator

$$
T v(x):=v(x)+\int_{-x}^{x} K(x, s) v(s) d s .
$$

The operator $T$ is called transmutation operator. Moreover, the function $K$ is not unique and can be chosen so that
$T[1]=f$ (see, e.g., $[7,8])$. When $q \in C^{1}[-b, b]$ such function $K$ is the unique solution of the Goursat problem

$$
\begin{aligned}
K_{x x}(x, s)-q(x) K(x, s) & =K_{s s}(x, s), \\
K(x, x) & =\frac{\alpha}{2}+\frac{1}{2} \int_{0}^{x} q(y) d y, \\
K(x,-x) & =\frac{\alpha}{2},
\end{aligned}
$$

For any $q \in C[-b, b]$ the kernel $K$ can be defined as $K(x, s)=$ $H((x+s) / 2,(x-s) / 2)$ and $|s| \leq|x| \leq b$, with $H$ being the unique solution of the Goursat problem

$$
\begin{aligned}
H_{u v}(u, v) & =q(u+v) H(u, v), \\
H(u, 0) & =\frac{\alpha}{2}+\frac{1}{2} \int_{0}^{u} q(s) d s, \\
H(0, v) & =\frac{\alpha}{2} .
\end{aligned}
$$

If the potential $q$ is $n$ times continuously differentiable on $(-b, b)$, the kernel $K(x, s)$ is $n+1$ times continuously differentiable with respect to both independent variables.

The following mapping property of the operator $T$ is used throughout the paper.

Proposition 2 (see [7]).

$$
T\left[x^{k}\right]=\varphi_{k}(x), \quad \forall k \in \mathbb{N}_{0} .
$$

The inverse operator $T^{-1}$ also has the form of a secondkind Volterra integral operator and satisfies the following correspondence of the initial values (see [8]):

$$
\begin{aligned}
v(0) & =u(0), \\
v^{\prime}(0) & =u^{\prime}(0)-\alpha u(0),
\end{aligned}
$$

where $v:=T^{-1} u$.

\section{The Noncharacteristic Cauchy Problem for (1)}

In this section an explicit solution of the noncharacteristic Cauchy problem for (1) in terms of the formal powers $\varphi_{k}$ is obtained. This fact is a direct consequence of the mapping property (9).

Definition 3 (see [1]). For the positive constants $\gamma_{1}, \gamma_{2}$, and $C_{1}$, the Holmgren class $H\left(\gamma_{1}, \gamma_{2}, C_{1}, t_{0}\right)$ is the set of infinitely differentiable functions $v$ defined on $\left|t-t_{0}\right|<\gamma_{2}$ that satisfy

$$
\left|v^{(j)}(t)\right| \leq C_{1} \gamma_{1}^{-2 j}(2 j) !, \quad j=0,1, \ldots
$$

for all $t \in\left|t-t_{0}\right|<\gamma_{2}$. 
Proposition 4. Let $q \in C[-b, b]$ and $u(x, t)$ be a solution of the noncharacteristic Cauchy problem

$$
\begin{array}{rlrl}
u_{x x}(x, t)-q(x) u(x, t) & =u_{t}(x, t), & & \\
& -b<x<b,|t|<\tau \\
u(0, t) & =F(t), & & |t|<\tau \\
u_{x}(0, t) & =G(t), & |t|<\tau
\end{array}
$$

where $F, G \in H(b, \tau, C, 0), C>0$. Then the series

$$
\begin{aligned}
\sum_{j=0}^{\infty} & {\left[\frac{F^{(j)}(t)}{(2 j) !}\left(\varphi_{2 j}(x)-\frac{\alpha}{2 j+1} \varphi_{2 j+1}(x)\right)\right.} \\
& \left.+\frac{G^{(j)}(t)}{(2 j+1) !} \varphi_{2 j+1}(x)\right]
\end{aligned}
$$

converges uniformly and absolutely for $|x| \leq r<b$ to the solution $u(x, t)$, where $\varphi_{k}$ are the formal powers (4).

Remark 5. Note that the functions $\varphi_{2 j}(x)-(\alpha / 2 j+$ 1)) $\varphi_{2 j+1}(x)$ coincide with the formal powers (4) constructed starting with the particular solution $g$ of (2) satisfying the initial conditions $g(0)=1$ and $g^{\prime}(0)=0$; see [9, Proposition 4.7].

Proof. Let $u(x, t)$ be a solution of (12). Consider the function $h:=T^{-1} u$, where the operator $T^{-1}$ is applied with respect to the variable $x$. The function $h$ is a solution of the heat equation (cf. [4, Theorem 2.1.2]) and due to (10) satisfies the following noncharacteristic Cauchy problem:

$$
\begin{aligned}
h_{x x} & =h_{t}, \quad-b<x<b, \quad|t|<\tau \\
h(0, t) & =F(t), \quad|t|<\tau \\
h_{x}(0, t) & =G(t)-\alpha F(t), \quad|t|<\tau .
\end{aligned}
$$

Since $G-\alpha F \in H(b, \tau,(1+\alpha) C, 0)$, the solution of this problem is given by the absolutely and uniformly convergent series for $|x| \leq r<b$ (see, e.g., [1]):

$$
\begin{aligned}
& h(x, t) \\
& \quad=\sum_{k=0}^{\infty}\left(\frac{F^{(k)}(t)}{(2 k) !} x^{2 k}+\frac{G^{(k)}(t)-\alpha F^{(k)}(t)}{(2 k+1) !} x^{2 k+1}\right) .
\end{aligned}
$$

Due to (9) we obtain

$$
\begin{aligned}
& u(x, t)=T h(x, t) \\
& =\sum_{k=0}^{\infty}\left[\frac{F^{(k)}(t)}{(2 k) !}\left(\varphi_{2 k}(x)-\frac{\alpha}{2 k+1} \varphi_{2 k+1}(x)\right)\right. \\
& \left.+\frac{G^{(k)}(t)}{(2 k+1) !} \varphi_{2 k+1}(x)\right] .
\end{aligned}
$$

This series converges uniformly and absolutely for $|x| \leq r<b$ due to the uniform boundedness of $T$ and of its inverse.

\section{Transmuted Heat Polynomials}

In this section a complete system of solutions of (1) is presented.

Consider the heat polynomials (see, e.g., [10]) defined by

$$
h_{n}(x, t)=n ! \sum_{k=0}^{[n / 2]} \frac{t^{k} x^{n-2 k}}{k !(n-2 k) !}, \quad n \in \mathbb{N}_{0} .
$$

Due to (9) we obtain that the functions

$$
u_{n}(x, t)=n ! \sum_{k=0}^{[n / 2]} \frac{t^{k} \varphi_{n-2 k}(x)}{k !(n-2 k) !}, \quad n \in \mathbb{N}_{0}
$$

are solutions of (1) for all $-b<x<b$ and $t>0$. Indeed, we have that $u_{n}=T h_{n}, n \in \mathbb{N}_{0}$, and

$$
\begin{gathered}
\frac{\partial^{2} u_{n}}{\partial x^{2}}-q(x) u_{n}(x, t)-\frac{\partial u_{n}}{\partial t}(x, t) \\
=T\left(\frac{\partial^{2} h_{n}}{\partial x^{2}}-\frac{\partial h_{n}}{\partial t}(x, t)\right)=0 .
\end{gathered}
$$

The completeness of the system of the heat polynomials with respect to the maximum norm proved in [4] and the uniform boundedness of $T$ and $T^{-1}$ imply the completeness of (20) in the space of classical solutions of (1). Thus, the following statement is true.

Theorem 6. Let $u(x, t)$ be continuous in $\bar{\Omega}$ and satisfy (1) in $\Omega$. Then given $\varepsilon>0$ there exist $N \in \mathbb{N}$ and constants $a_{0}, a_{1}, \ldots, a_{N}$ such that

$$
\max _{\bar{\Omega}}\left|u(x, t)-\sum_{n=0}^{N} a_{n} u_{n}(x, t)\right|<\varepsilon .
$$

Proof. Choose $\varepsilon>0$. Consider $h(x, t)=T^{-1} u(x, t)$. Due to the completeness of (19), for any $\varepsilon_{1}>0$ there exist $N \in \mathbb{N}$ and constants $a_{0}, a_{1}, \ldots, a_{N}$ such that

$$
\max _{\bar{\Omega}}\left|h(x, t)-\sum_{n=0}^{N} a_{n} h_{n}(x, t)\right|<\varepsilon_{1} .
$$

Then

$$
\begin{aligned}
& \max _{\bar{\Omega}}\left|u(x, t)-\sum_{n=0}^{N} a_{n} u_{n}(x, t)\right| \\
& \quad=\max _{\bar{\Omega}}\left|\operatorname{Th}(x, t)-\sum_{n=0}^{N} a_{n} T h_{n}(x, t)\right| \leq C \varepsilon_{1},
\end{aligned}
$$

where the constant $C$ is the uniform norm of $T$. The choice of $\varepsilon_{1}=\varepsilon / C$ finishes the proof.

\section{Solution of Initial Boundary Value Problems for (1)}

Consider the problem to find the solution of the equation

$$
u_{x x}(x, t)-q(x) u(x, t)=u_{t}(x, t), \quad(x, t) \in \Omega
$$


subject to the Dirichlet boundary conditions

$$
\begin{gathered}
u(-b, t)=\psi_{1}(t), \\
u(b, t)=\psi_{2}(t),
\end{gathered}
$$

$$
t \in[0, \tau]
$$

and the initial condition

$$
u(x, 0)=\varphi(x), \quad x \in[-b, b]
$$

where $\psi_{1}, \psi_{2}$, and $\varphi$ are continuously differentiable functions satisfying the compatibility conditions

$$
\begin{aligned}
& \psi_{1}(0)=\varphi(-b), \\
& \psi_{2}(0)=\varphi(b) .
\end{aligned}
$$

Problem (25)-(27) possesses a unique solution which depends continuously on the data (see, e.g., [11]).

The result of Theorem 6 suggests the following simple method to approximate the solution of problem (25)-(27). The approximate solution $\tilde{u}$ is sought in the form

$$
\tilde{u}(x, t)=\sum_{n=0}^{N} a_{n} u_{n}(x, t) .
$$

Since every $u_{n}$ is a solution of (25), their linear combination satisfies (25) as well. The coefficients $\left\{a_{n}\right\}_{n=0}^{N}$ are sought in such a way that $\widetilde{u}$ satisfy the initial and the boundary conditions approximately. For this we used the collocation method. $M$ points $\left\{\left(x_{i}, t_{i}\right)\right\}_{i=1}^{M}$ are chosen on the parabolic boundary $(\{-b\} \times[0, \tau]) \cup([-b, b] \times\{0\}) \cup(\{b\} \times[0, \tau])$ in order to construct a linear system of equations for the coefficients $\left\{a_{n}\right\}_{n=0}^{N}$ :

$$
\sum_{n=0}^{N} a_{n} u_{n}\left(x_{i}, t_{i}\right)= \begin{cases}\psi_{1}\left(t_{i}\right), & x_{i}=-b \\ \varphi\left(x_{i}\right), & t_{i}=0, \\ \psi_{2}\left(t_{i}\right), & x_{i}=b \\ & i=1, \ldots, M .\end{cases}
$$

System (30) is the result of imposing conditions (26) and (27) onto the approximate solution (29). Using the pseudoinverse matrix system (30) is solved, and the approximate solution (29) is computed on $\Omega$ using the obtained coefficients $\left\{a_{n}\right\}_{n=0}^{N}$ and the definition of the transmuted heat polynomials (20).

Needless to add, the same approach is applicable to other kinds of boundary conditions.

\section{Numerical Illustration}

We present a numerical example of the application of the method described in the previous section. It reveals a remarkable accuracy with very little computational efforts. The implementation was realized in MATLAB 2012.

On the first step a nonvanishing solution $f$ of (2) was computed using the SPPS method (see [3]). The

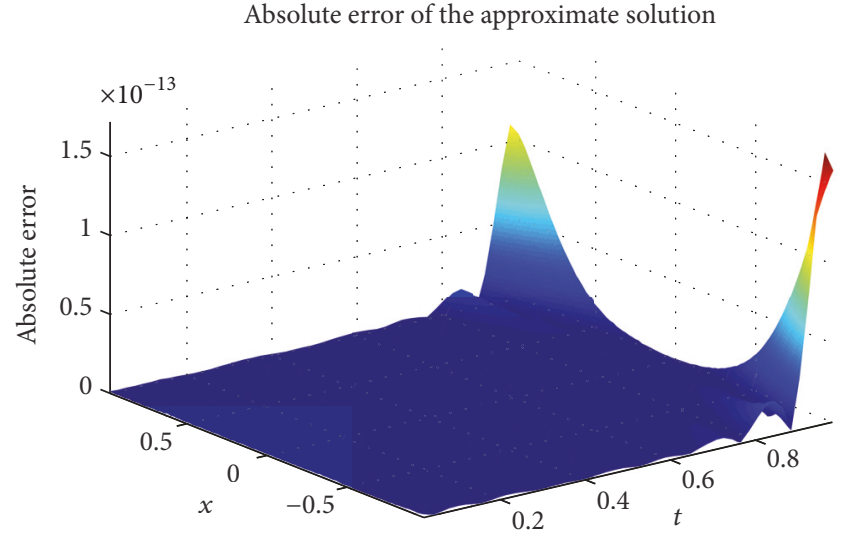

FIgURE 1: The absolute value of the difference $\left|u(x, t)-u_{26}(x, t)\right|$ between the exact and the approximate solutions for problem (31)-(33).

formal powers $\varphi_{k}$ were constructed like in [6] using the spapi and fnint MATLAB routines from the spline Toolbox. Then the transmuted heat polynomials (20) were calculated. In order to obtain a unique solution of system (30) $N+1$ equally spaced points on the parabolic boundary were chosen. Finally, the approximate solution (29) was computed on a mesh of $200 \times 100$ points in the interior of the rectangle and compared with the corresponding exact solution.

Example 1. Consider the initial Dirichlet problem

$$
\begin{aligned}
& u_{x x}(x, t)-x^{2} u(x, t)=u_{t}(x, t), \\
& \qquad(x, t) \in(-1,1) \times(0,1), \\
& u(x, 0)=e^{-0.5 x^{2}}, \quad x \in[-1,1], \\
& u(-1, t)=u(1, t)=e^{-0.5-t}, \quad t \in[0,1] .
\end{aligned}
$$

The exact solution of this problem has the form

$$
u(x, t)=\exp \left(-\frac{1}{2} x^{2}-t\right)
$$

The distribution of the absolute error of the approximate solution for $N=26$ is presented on Figure 1. The maximum absolute error of the approximate solution is of order $10^{-13}$.

It is often stated that boundary collocation methods (in particular, the heat polynomials method) lead to illconditioned systems of linear equations; see [12-14]. It is also the case for the proposed method. As is illustrated in Table 1, the condition number of the matrix in (30) grows rather fast. Nevertheless, the straightforward implementation of the proposed method presented no numerical difficulties. The convergence and the robustness of the method are illustrated in Table 1 where the maximum absolute and the maximum relative error of the approximate solution for different values of $N$ used for approximation (29) are presented. As one can appreciate, the convergence rate for small values of $N$ is exponential, while values of $N$ considerably larger than the optimum do not lead to significant loss of precision. 
TABLE 1: Maximal absolute and relative errors of the approximate solution and condition number of the matrix in (30) for problem (31)-(33) obtained for different values of $N$ in (29).

\begin{tabular}{lccc}
\hline$N$ & Max. absolute error & Max. relative error & Cond. number \\
\hline 5 & $2.3 \cdot 10^{-2}$ & $6.2 \cdot 10^{-2}$ & 55.7 \\
10 & $1.4 \cdot 10^{-4}$ & $5.5 \cdot 10^{-4}$ & $1.82 \cdot 10^{5}$ \\
15 & $9.6 \cdot 10^{-8}$ & $4.0 \cdot 10^{-7}$ & $3.65 \cdot 10^{9}$ \\
20 & $2.0 \cdot 10^{-10}$ & $8.6 \cdot 10^{-10}$ & $1.76 \cdot 10^{14}$ \\
23 & $7.6 \cdot 10^{-13}$ & $3.2 \cdot 10^{-12}$ & $1.67 \cdot 10^{17}$ \\
26 & $1.8 \cdot 10^{-13}$ & $7.8 \cdot 10^{-13}$ & $2.59 \cdot 10^{23}$ \\
29 & $2.5 \cdot 10^{-12}$ & $1.1 \cdot 10^{-11}$ & $1.23 \cdot 10^{23}$ \\
34 & $1.7 \cdot 10^{-10}$ & $7.3 \cdot 10^{-10}$ & $1.37 \cdot 10^{25}$ \\
39 & $2.3 \cdot 10^{-9}$ & $9.8 \cdot 10^{-9}$ & $2.86 \cdot 10^{29}$ \\
50 & $4.7 \cdot 10^{-10}$ & $2.1 \cdot 10^{-9}$ & $1.25 \cdot 10^{41}$ \\
75 & $6.1 \cdot 10^{-11}$ & $2.7 \cdot 10^{-10}$ & $2.89 \cdot 10^{73}$ \\
100 & $2.8 \cdot 10^{-10}$ & $1.2 \cdot 10^{-9}$ & $5.63 \cdot 10^{105}$ \\
\hline
\end{tabular}

Moreover, a simple test based on the accuracy of fulfilment of the initial and boundary conditions (32)-(33) can be utilized to estimate both the optimal $N$ and the accuracy of the obtained approximate solution.

\section{Conclusions}

A complete system of solutions of equation (1) is obtained. The solutions represent the images of the heat polynomials under the action of the transmutation operator. They are shown to be convenient for uniform approximation of solutions of initial boundary value problems for (1) as well as for explicit solution of the noncharacteristic Cauchy problem. Besides the Dirichlet boundary conditions considered in this paper the method is applicable to other standard boundary conditions. The complete system of solutions obtained can be used for solving moving and free boundary problems.

\section{Conflicts of Interest}

The authors declare that there are no conflicts of interest regarding the publication of this paper.

\section{Acknowledgments}

Research was supported by CONACYT, Mexico, via Project 222478.

\section{References}

[1] J. R. Cannon, The One-Dimensional Heat Equation, AddisonWesley, Reading, Mass, USA, 1984.

[2] V. V. Kravchenko, "A representation for solutions of the SturmLiouville equation," Complex Variables and Elliptic Equations. An International Journal, vol. 53, no. 8, pp. 775-789, 2008.

[3] V. V. Kravchenko and R. M. Porter, "Spectral parameter power series for Sturm-Liouville problems," Mathematical Methods in the Applied Sciences, vol. 33, no. 4, pp. 459-468, 2010.

[4] D. L. Colton, Solution of Boundary Value Problem by The Method of Integral Operator, Pitman Publishing, San Francisco, Calif, USA, 1976.
[5] V. A. Marchenko, Sturm-Liouville Operators and Applications, vol. 22 of Operator Theory: Advances and Applications, Birkhäuser, Basel, Switzerland, 1986.

[6] K. V. Khmelnytskaya, V. V. Kravchenko, S. M. Torba, and S. Tremblay, "Wave polynomials, transmutations and Cauchy's problem for the Klein-Gordon equation," Journal of Mathematical Analysis and Applications, vol. 399, no. 1, pp. 191-212, 2013.

[7] H. M. Campos, V. V. Kravchenko, and S. . Torba, “Transmutations, L-bases and complete families of solutions of the stationary Schrödinger equation in the plane," Journal of Mathematical Analysis and Applications, vol. 389, no. 2, pp. 1222-1238, 2012.

[8] V. V. Kravchenko and S. M. Torba, "Transmutations for Darboux transformed operators with applications," Journal of Physics A: Mathematical and Theoretical, vol. 45, no. 7, Article ID 075201, 2012.

[9] V. V. Kravchenko and S. M. Torba, "Transmutations and Spectral Parameter Power Series in Eigenvalue Problems," in Operator Theory, Pseudo-Differential Equations, and Mathematical Physics, vol. 228 of Operator Theory: Advances and Applications, pp. 209-238, Birkhäuser, Basel, Switzerland, 2013.

[10] P. C. Rosenbloom and D. V. Widder, "Expansions in terms of heat polynomials and associated functions," Transactions of the American Mathematical Society, vol. 92, pp. 220-266, 1959.

[11] V. S. Vladimirov, Equations of Mathematical Physics, Mir Publishers, Moscow, Russia, 2nd edition, 1983.

[12] S. r. Christiansen and P. C. Hansen, "The effective condition number applied to error analysis of certain boundary collocation methods," Journal of Computational and Applied Mathematics, vol. 54, no. 1, pp. 15-36, 1994.

[13] J. A. Kołodziej and A. P. Zielin'ski, Boundary Collocation Techniques and their Application in Engineering, WIT Press, Southampton, Boston, 2009.

[14] Z.-C. Li, T.-T. Lu, H.-Y. Hu, and A. H.-D. Cheng, Trefftz and Collocation Methods, WIT Press, Southampton, 2008. 


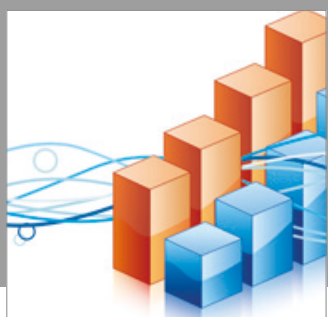

Advances in

Operations Research

vatersals

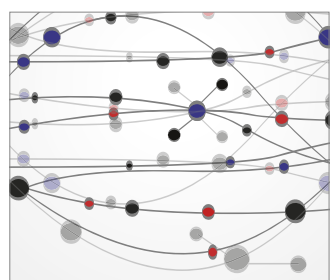

\section{The Scientific} World Journal
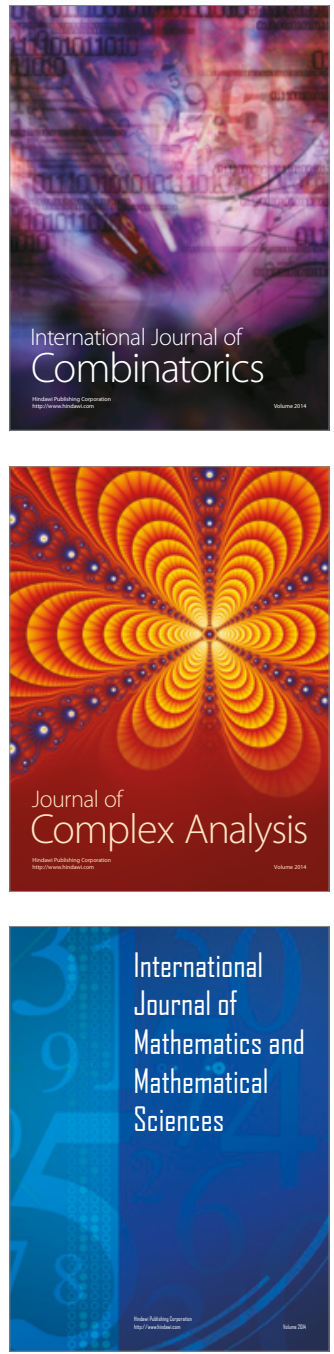
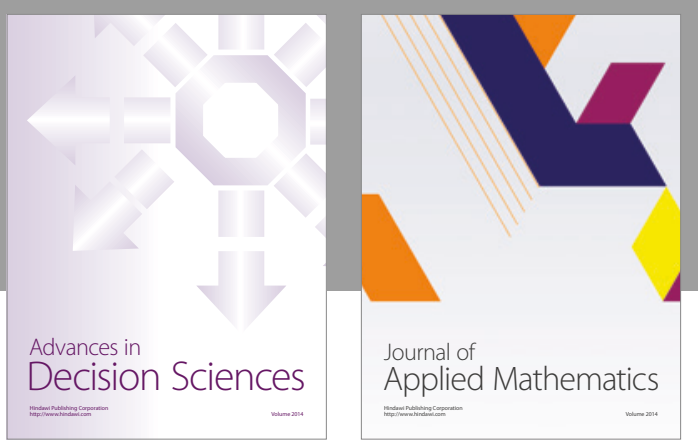

Algebra

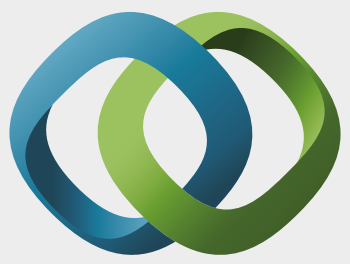

\section{Hindawi}

Submit your manuscripts at

https://www.hindawi.com
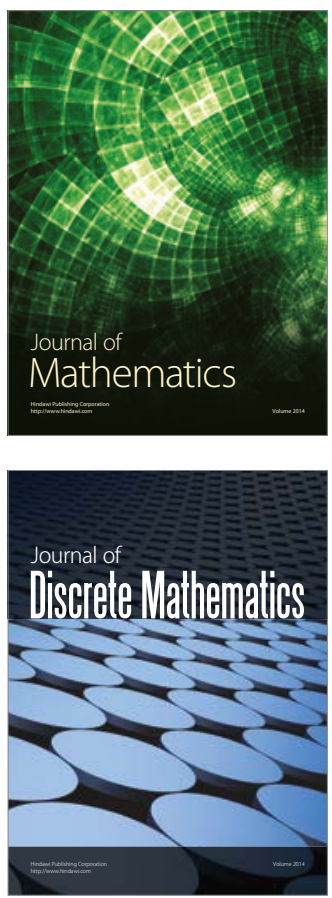

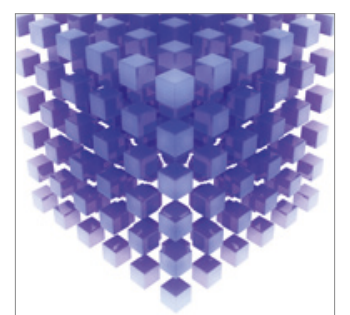

Mathematical Problems in Engineering
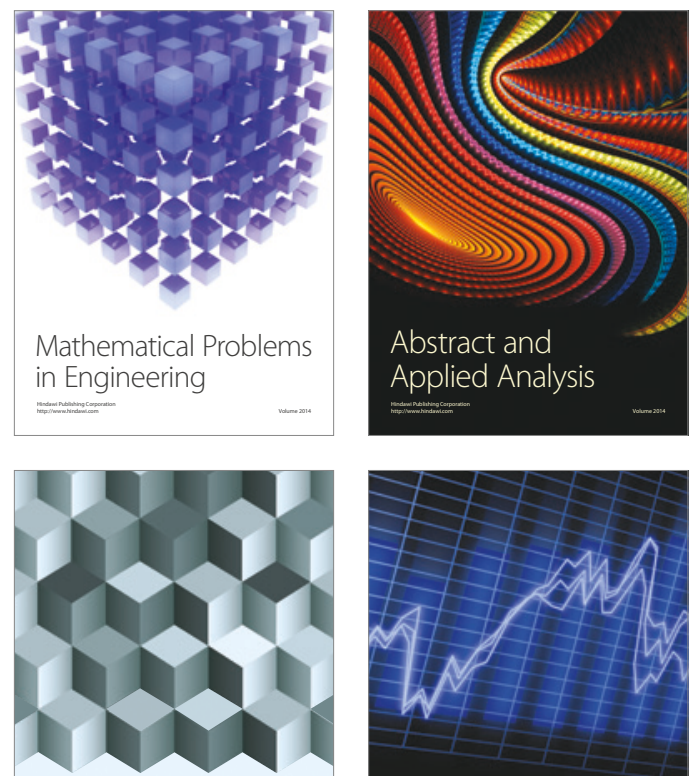

Journal of

Function Spaces

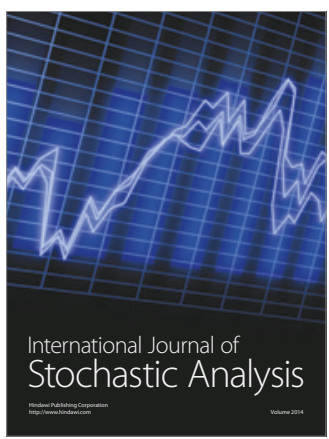

Probability and Statistics
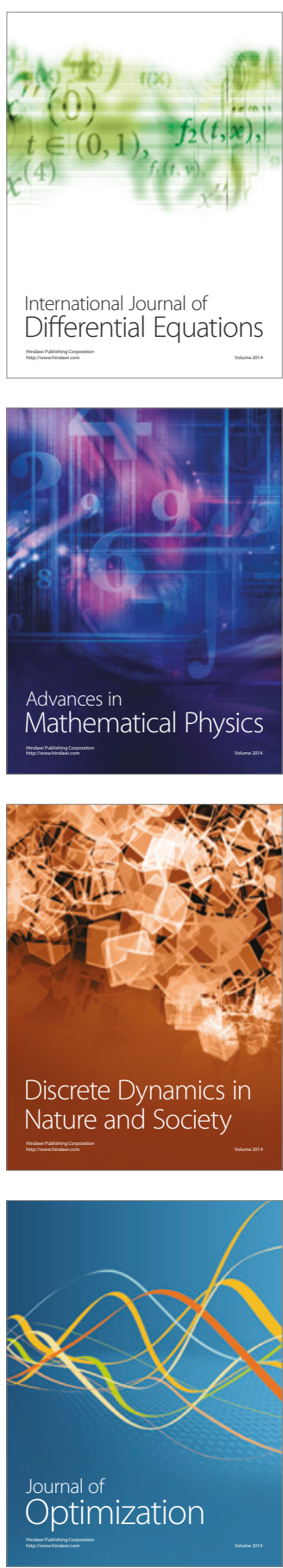